\title{
Disfunción ventricular subclínica por Speckle Tracking en pacientes adultos con coartación de aorta
}

\author{
Nilda Espinola-Zavaleta1, José Carlos Rúan-Díaz', María Elena Soto², Nydia Ávila-Vanzzini', Guillermo \\ Bracamontes-Castelo ${ }^{3}$ y Leonel Avendaño-Pérez ${ }^{4}$ \\ ${ }^{1}$ Laboratorio de Ecocardiografía y Hemodinámica no-invasiva; ${ }^{2}$ Departamento de Inmunología; ${ }^{3}$ Verano de Investigación; ${ }^{4}$ Servicio Social en \\ Investigación, Instituto Nacional de Cardiología Ignacio Chávez, Ciudad de México, México
}

\section{Resumen}

Objetivo: Buscar la presencia de disfunción sistólica temprana del ventrículo izquierdo (VI) en pacientes adultos con coartación aórtica (CoAo) e hipertensión arterial sistémica (HAS), comparados con pacientes hipertensos sistémicos sin CoAo y controles sanos, mediante Speckle Tracking. Métodos: Se estudiaron 61 sujetos que acudieron en forma consecutiva a consulta externa. A todos se les realizó historia clínica, electrocardiograma de reposo y ecocardiograma convencional y con Speckle Tracking. Resultados: 15 pacientes con CoAo y HAS estuvieron en clase funcional de la New York Heart Association (NYHA) I, y cinco en clase funcional NYHA II. Se observó una correlación inversa significativa entre la deformación longitudinal global y el índice de masa del VI en el grupo de CoAo y HAS, así como en los pacientes hipertensos sistémicos ( $r=0.53$, $p=0.02 ; r=0.52, p<0.0001$, respectivamente). Conclusiones: En los pacientes con CoAo y HAS, la deformación longitudinal global y el índice de masa ventricular izquierda se relacionan significativamente con disfunción subclínica del VI. Estos parámetros son importantes y deben ser tomados en cuenta para normar la conducta terapéutica oportuna.

PALABRAS CLAVE: Coartación de aorta. Deformación longitudinal. Deformación radial. Ecocardiografía. Speckle Tracking.

\begin{abstract}
Objective: The aim of this study was to investigate the presence of early left ventricular (LV) systolic dysfunction in adult patients with aortic coarctation (AoCo) and systemic arterial hypertension (SAH) compared to systemic hypertensive patients without coarctation and healthy controls by speckle tracking. Methods: Sixty-one subjects were studied, who attended consecutively to external consultation. All were submitted to clinical history, resting electrocardiogram, conventional echocardiogram and with Speckle Tracking. Results: 15 patients with AoCo and SAH were in functional class NYHA I, and five in functional class NYHA II. A significant inverse correlation was observed between the global longitudinal deformation and the left ventricular mass index in the AoCo and SAH group and in the hypertensive patients $(r=0.53, p=0.02 ; r=0.52, p<0.0001$, respectively). Conclusions: In patients with AoCo and SAH, global longitudinal deformation and left ventricular mass index are significantly related to subclinical $L V$ dysfunction. These parameters are important and should be taken into account to regulate timely therapeutic behavior.
\end{abstract}

KEY WORDS: Coarctation of the aorta. Longitudinal deformation. Radial deformation. Echocardiography. Speckle Tracking.

\author{
Correspondencia: \\ Nilda Espinola-Zavaleta \\ Juan Badiano, 1 \\ Col. Sección XVI, \\ Fecha de recepción: 07-08-2017 \\ Ciudad de México, México \\ E-mail: niesza2001@ hotmail.com \\ Gac Med Mex. 2017;153;Sup 2:S18-S26 \\ Contents available at PubMed \\ DOI://dx.doi.org/10.24875/GMM.M17000003
}




\section{Introducción}

La coartación aórtica (CoAo) es una cardiopatía congénita que tiene una prevalencia del 5 al $8 \%$ de todos los defectos congénitos del corazón ${ }^{1-4}$. En el $85 \%$ de los casos se asocia con aorta bicúspide y en el 3 al 10\% con estenosis subvalvular, valvular o supravalvular aórtica ${ }^{4}$. La forma aislada se reporta en $3 / 10,000$ nacidos vivos ${ }^{4}$ y en 1:1550 hallazgos de necropsias, y más frecuente en hombres $(1.5: 1)^{5}$. Esta cardiopatía se debe a la anormalidad en el desarrollo del cuarto y el sexto arcos aórticos, por la migración de células del músculo liso del conducto arterioso hacia la aorta periductal, y se define como un estrechamiento de la aorta, habitualmente en la proximidad del ligamento arterioso, adyacente al nacimiento de la subclavia izquierda ${ }^{4-6}$ (Fig. 1).

El $80 \%$ de los pacientes con esta cardiopatía presenta hipertensión arterial sistémica (HAS). La CoAo produce una poscarga incrementada del ventrículo izquierdo (VI), lo que resulta en mayor tensión parietal, hipertrofia compensatoria y disfunción sistólica y diastólica del $\mathrm{VI}^{4,7,8}$.

El ecocardiograma es una técnica no invasiva que permite caracterizar la CoAo y su repercusión hemodinámica al VI (hipertrofia, dilatación y disfunción ventricular valorada por la fracción de expulsión del VI $[\mathrm{FEVI}])^{9}$. Actualmente se conoce que la FEVI es un marcador subóptimo de disfunción ventricular sistólica en etapas incipientes de daño miocárdico. El Speckle Tracking es una técnica sofisticada de ecocardiografía que proporciona una evaluación cuantitativa del movimiento del tejido miocárdico, independientemente del ángulo y la geometría ventricular, que permite detectar la disfunción miocárdica subclínica del $\mathrm{V}^{10-13}$.

El objetivo de este estudio fue buscar la presencia de disfunción sistólica temprana del VI en pacientes adultos con CoAo y HAS comparados con pacientes hipertensos sistémicos sin CoAo y controles sanos, mediante la técnica ecocardiográfica de Speckle Tracking.

\section{Métodos}

Entre marzo de 2016 y febrero de 2017 se realizó un estudio comparativo, observacional, prospectivo, retrolectivo, descriptivo y analítico, en el cual se evaluaron 61 sujetos que acudieron en forma consecutiva a consulta externa del Instituto Nacional de Cardiología Ignacio Chávez: 21 con CoAo y HAS, 20 pacientes con HAS de más de 5 años de evolución, sin CoAo, y 20 controles sanos.

Se incluyeron pacientes mayores de 18 años con CoAo y HAS definida por una presión sistólica y diastólica $\geq 140 / 90 \mathrm{mmHg}$ de acuerdo con las recomendaciones de la Sociedad de Hipertensión ${ }^{14}$, y pacientes con CoAo y HAS sin lesión valvular aórtica o con insuficiencia y/o estenosis valvular aórtica ligera.

Se excluyó a todos los pacientes con CoAo y presión arterial sistémica normal, a aquellos que tuvieron estenosis y/o insuficiencia moderada a importante, $y$ a los que se rehusaron a participar en el estudio.

A todos los pacientes se les realizó historia clínica completa, electrocardiograma de reposo de

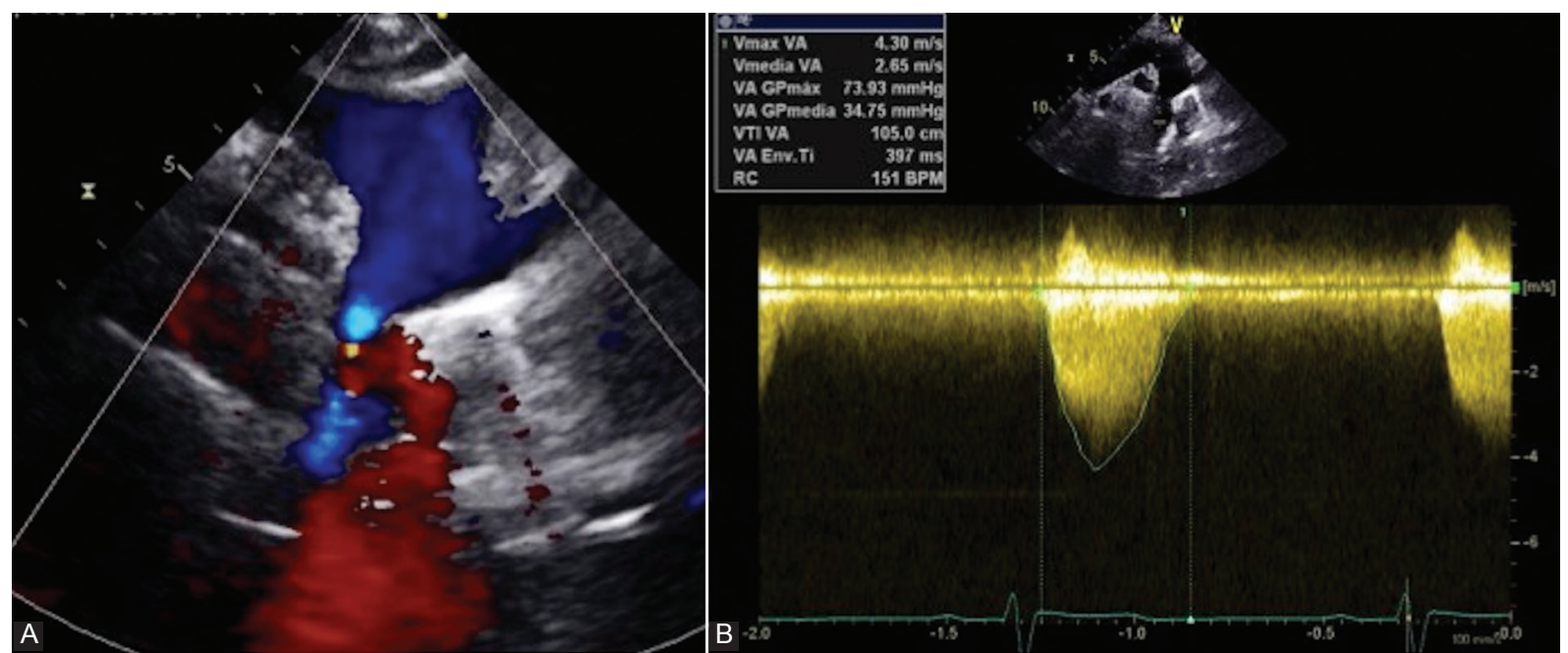

Figura 1. Ecocardiograma transtorácico bidimensional y con Doppler color (A) y Doppler continuo (B) en la vista supraesternal, que muestra el sitio de la CoAo, la velocidad máxima y los gradientes máximo y medio transcoartación. 
12 derivaciones y ecocardiograma convencional para determinar el índice de masa del VI, la función sistólica y la función diastólica con la relación E/A del flujo mitral y la relación E/e' para valorar las presiones de llenado del VI. En el eje supraesternal se valoró la gravedad de la CoAo colocando la muestra de volumen del Doppler continuo en el sitio de la CoAo para determinar la velocidad máxima y el gradiente máximo y medio transcoartación, en $\mathrm{cm} / \mathrm{s}$ y $\mathrm{mmHg}$, respectivamente.

La deformación longitudinal, circunferencial y radial, así como el twist y la torsión del VI, se valoraron mediante el método de Speckle Tracking.

\section{Estudio ecocardiográfico}

Los estudios ecocardiográficos transtorácicos convencionales se realizaron utilizando un equipo comercialmente disponible (Vivid 9, GE Vingmed Ultrasound, Horten, Noruega), con una sonda matricial M3S. Se obtuvieron los diámetros ventriculares en telediástole y en telesístole, así como el grosor del tabique interventricular y de la pared posterior de acuerdo con las guías de la Sociedad Americana de Ecocardiografia ${ }^{9}$. La masa ventricular izquierda se midió con la fórmula de área-longitud y se indexó para el área de la superficie corporal. Se consideró hipertrofia cuando el índice de masa era $>95 \mathrm{~g} / \mathrm{m}^{2}$ en las mujeres y $>115 \mathrm{~g} / \mathrm{m}^{2}$ en los hombres ${ }^{9,15}$. La FEVI se calculó utilizando el método biplano de Simpson. La velocidad máxima y los gradientes máximo y medio transcoartación en el sitio de la CoAo fueron evaluados con Doppler continuo en el plano supraesternal. La función diastólica se determinó con Doppler pulsado en el plano apical de cuatro cámaras, colocando la muestra de volumen en la punta de las valvas mitrales para medir las velocidades de onda $E$ y onda $A$, y la relación $E / A$ (disfunción diastólica cuando E/A $<0.80 \geq 2$ ). La velocidad de la onda e' septal se midió con Doppler tisular, colocando la muestra de volumen en la porción basal del tabique interventricular. Cuando la relación $\mathrm{E} / \mathrm{e}>14$, se consideró que había incremento en las presiones de llenado del VI.

\section{Speckle Tracking}

Los pacientes se evaluaron en decúbito lateral izquierdo, utilizando un ecocardiógrafo con software para mecánica ventricular. Se obtuvieron proyecciones bidimensionales en los planos apicales de cuatro, tres y dos cámaras, y en los ejes cortos paraesternales a nivel de la válvula mitral, los músculos papilares y apical. Todas las imágenes se obtuvieron con una frecuencia de fotogramas entre 50 y 80 cuadros/s. Los ejes cortos del VI tuvieron forma circular para evaluar la deformación en las direcciones radial y circunferencial anatómicamente correctas ${ }^{10-12}$. Todos los exámenes fueron enviados a la estación de trabajo para su análisis posterior.

El límite del endocardio del VI se trazó de acuerdo con las especificaciones del programa. Se utilizaron las tres proyecciones apicales (cuatro, tres y dos cámaras) para la determinación de la deformación longitudinal (Fig. 2). Para la deformación radial y circunferencial se utilizó el eje corto a nivel de los músculos papilares, y la rotación se determinó en los ejes cortos paraesternales a nivel de la válvula mitral (basal) y a nivel apical. El programa divide automáticamente cada plano ecocardiográfico en seis segmentos y realiza el análisis de la deformación fotograma a fotograma, proporciona una confirmación automatizada del contorno y genera los valores de deformación, expresados en porcentaje. Los valores de la deformación longitudinal global y segmentaria se presentan en un mapa polar (ojo de buey).

La rotación es el desplazamiento angular promedio de la capa media del VI. Durante la sístole, la base rota en sentido horario y el ápex lo hace en sentido antihorario. La rotación en sentido antihorario se expresa con valores positivos cuando se visualiza desde el vértice, y la rotación en sentido horario se expresa con valores negativos y en grados.

El twist es la diferencia en la rotación del VI entre el ápex y la base respecto a un eje central imaginario. Se obtiene restando a la rotación apical la rotación basal, y el resultado se expresa en grados (Fig. 3). La torsión es el ángulo de rotación del eje largo del VI normalizada a la longitud total del ventrículo, y se expresa en grados por centímetro.

Los valores normales de la deformación longitudinal global son $\geq-20 \%$, los de la deformación circunferencial son $\geq-25 \%$, y los de la deformación radial son $\geq 40 \%$. El valor normal del twist es de 9.9 \pm 4.1 grados, y el de la rotación es de $1.35 \mathrm{grados} / \mathrm{cm}$ \pm 0.54 .

\section{Análisis estadístico}

Las variables numéricas fueron evaluadas con una distribución gaussiana con mediciones de tendencia central, considerando la media y la desviación estándar, y en las variables categóricas se hizo con 


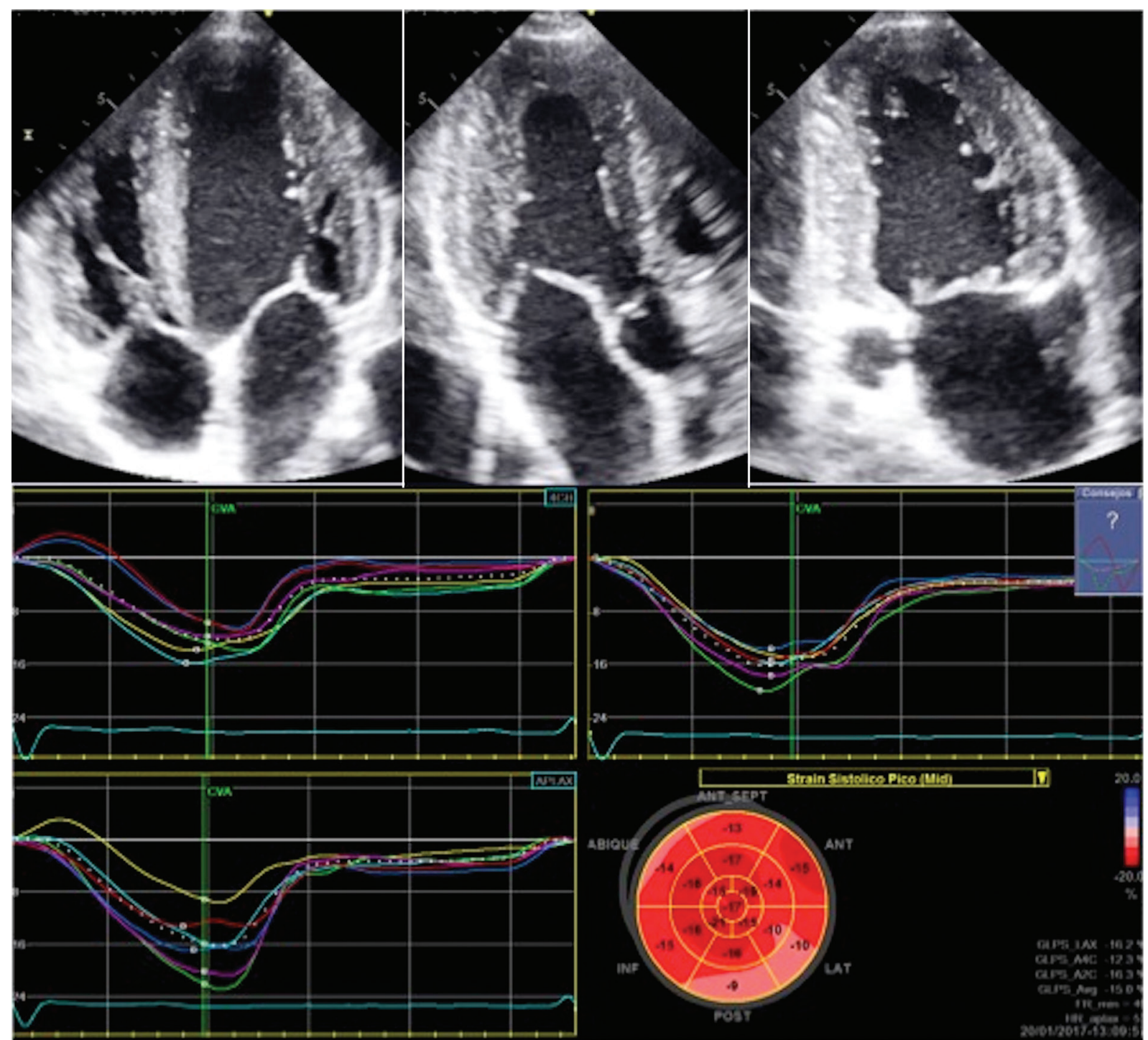

Figura 2. Determinación de la deformación longitudinal por Speckle Tracking en los planos apicales de cuatro, tres y dos cámaras, y su representación gráfica. El ojo de buey muestra la deformación longitudinal global y segmentaria del ventrículo izquierdo.

porcentajes. El análisis bivariado se ajustó según la distribución. Se utilizaron las pruebas $t$ de Student 0 U de Mann-Whitney con variables categóricas, así como la de ji al cuadrado con la corrección de Yates o la prueba exacta de Fisher. Se realizó una comparación de medias entre grupos mediante Kruskall Wallis, y las correlaciones entre variables mediante correlación de Pearson.

Se evaluó la reproducibilidad intraobservador e interobservador en 10 sujetos elegidos al azar. Se expresó como porcentaje del error, derivado como la diferencia absoluta entre dos conjuntos de mediciones dividido por la media de las observaciones, y usando coeficientes de correlación intraclase. Las medidas intraobservador se realizaron con 15 días de diferencia. Para calcular la variabilidad entre observadores, el segundo observador experimentado (N.A.), que estaba cegado a los hallazgos del primer observador, analizó los conjuntos de datos bidimensionales de Speckle Tracking.

Todos los pacientes firmaron un consentimiento informado.

\section{Resultados}

Se estudiaron 61 sujetos, 33 mujeres (54\%) y 28 hombres (46\%), con edad promedio de 32 (18-77) años. Las características demográficas y clínicas de los grupos estudiados se muestran en la tabla 1.

Quince pacientes con CoAo y HAS se encontraban en clase funcional I de la New York Heart Association (NYHA), y cinco en clase funcional NYHA II; de los 


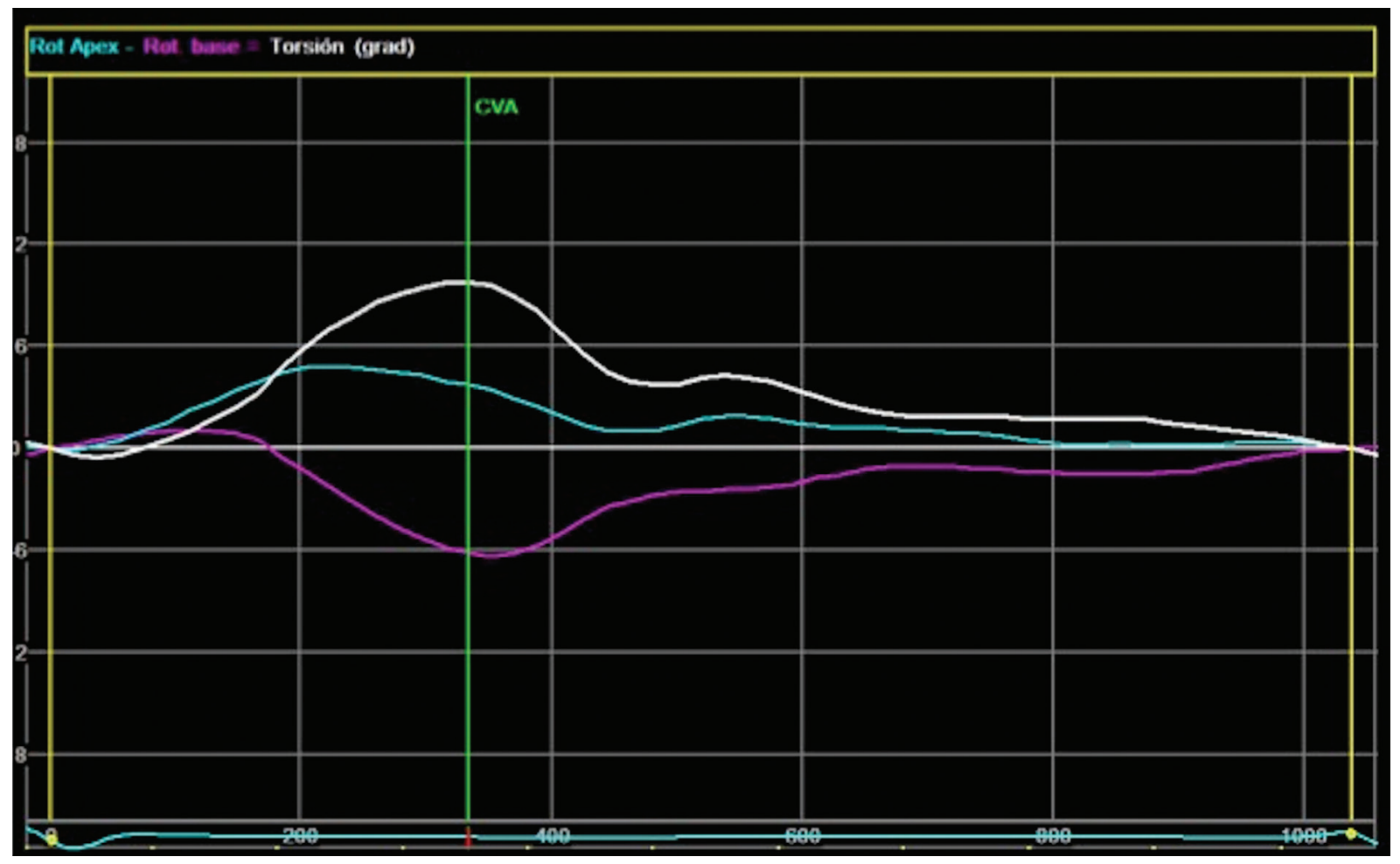

Figura 3. Representación gráfica del twist y la torsión del ventrículo izquierdo en un paciente con coartación de aorta.

pacientes con HAS, 19 estaban en clase funcional NYHA I y uno en clase funcional NYHA II. En el electrocardiograma de reposo, 17 pacientes con CoAo tuvieron hipertrofia ventricular izquierda y seis de ellos con sobrecarga sistólica; de los pacientes con HAS, 14 tuvieron hipertrofia ventricular izquierda. En la radiografía de tórax se encontró signo de Roesler en el $100 \%$ de los pacientes con CoAo.

En la figura 4 se muestran los tipos de CoAo, siendo la preductal la más frecuente. Las características ecocardiografías se muestran en la tabla 2. Se encontraron diferencias estadísticamente significativas en el volumen de la aurícula izquierda, el grosor parietal y los diámetros del VI, en el índice de masa ventricular izquierda, en la función sistólica y diastólica del VI, y en la presión sistólica de la arteria pulmonar. El 70\% (14/20) de los pacientes tuvieron aorta bivalva.

Los valores de la deformación longitudinal, radial y circunferencial, y de la rotación y del twist, se muestran en la tabla 3 y la figura 5 . Se observaron cambios significativos solo en la deformación longitudinal y radial del VI en los pacientes con CoAo y en el grupo con HAS. El twist y la rotación no mostraron significación estadística en ninguno de los grupos estudiados.

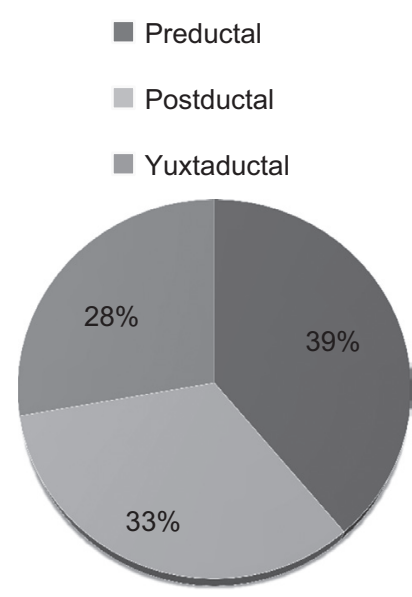

Figura 4. Gráfica que muestra el porcentaje de los diferentes tipos de coartación de aorta.

Se observó una correlación inversa significativa entre la deformación longitudinal global y el índice de masa del $\mathrm{VI}(r=0.51 ; p=0.0001)$ en el grupo total. Al analizarlo por subgrupos se encontró que, en el grupo de CoAo y en el grupo de pacientes hipertensos, esta correlación fue estadísticamente significativa, con $r=0.53(p=0.02)$ (Fig. 6) y $r=0.52(p<0.0001)$, respectivamente.

La correlación entre la deformación longitudinal y la FEVI del grupo total fue negativa, con $r=0.54$ 
Tabla 1. Características demográficas y clínicas del grupo estudiado

\begin{tabular}{|c|c|c|c|c|c|}
\hline & $\begin{array}{c}\text { Total } \\
n=61(100 \%)\end{array}$ & $\begin{array}{c}\text { Controles } \\
n=21(34 \%)\end{array}$ & $\begin{array}{c}\text { HAS } \\
n=20(33 \%)\end{array}$ & $\begin{array}{c}\text { CoAo } \\
n=20(33 \%)\end{array}$ & $\mathbf{p}$ \\
\hline Mujer & $33(57)$ & $8(24)$ & $14(42)^{*}$ & $11(33)$ & 0.06 \\
\hline Hombre & $28(43)$ & $13(46)^{\dagger}$ & $6(21)$ & $9(32)$ & 0.06 \\
\hline \multicolumn{6}{|l|}{ Promedio, mínimo-máximo } \\
\hline Edad (años) & $32(18-77)$ & $28 *(22-49)$ & $57(32-78)$ & $27(20-49)$ & 0.0001 \\
\hline Peso $(\mathrm{kg})$ & $72(49-103)$ & $73(53-93)$ & $75(52-103)$ & $67(49-80)$ & NS \\
\hline Talla $(m)$ & $1.64(1.42-1.95)$ & $1.69(1.51-1.95)$ & $1.55(1.42-1.69)$ & $1.62(1.43-1.80)$ & 0.001 \\
\hline Área de superficie corporal & $1.76(1.40-2.10)$ & $1.83(1.54-2.07)$ & $1.74(1.40-2.10)$ & $1.74(1.41-1.99)$ & 0.056 \\
\hline Presión arterial sistólica brazo izquierdo (mmHg) & $120(90-220)$ & $105(90-120)^{¥}$ & $140(110-185)$ & $130(110-220)$ & 0.001 \\
\hline Presión arterial diastólica brazo izquierdo (mmHg) & $75(60-102)$ & $70(60-80)^{¥}$ & $85(70-102)$ & $85(75-100)$ & 0.001 \\
\hline Presión arterial sistólica brazo derecho (mmHg) & $124(90-200)$ & $105(90-110)^{*}$ & $133(110-176)$ & $140(120-200)$ & 0.001 \\
\hline Presión arterial diastólica brazo derecho (mmHg) & $70(60-110)$ & $68(60-70)^{*}$ & $80(70-105)$ & $90(77-110)$ & 0.001 \\
\hline \multicolumn{6}{|l|}{ Síntomas } \\
\hline Disnea & $6(10)$ & 0 & 1 & $5^{*}$ & 0.06 \\
\hline Ángor & $3(5)$ & 0 & 0 & 3 & 0.06 \\
\hline Evento vascular cerebral & $1(2)$ & 0 & 0 & 1 & NS \\
\hline HAS & $38(62)$ & $0^{*}$ & $20(53)$ & $18(47)$ & 0.0001 \\
\hline
\end{tabular}

Análisis con Kruskall-Wallis, ji al cuadrado y prueba exacta de Fisher.

CoAo: coartación de aorta; HAS: hipertensión arterial sistémica; NS: no significativo. *: Control vs HAS; ¥: Control vs HAS and CoAo with the same statistical significant difference

Tabla 2. Características ecocardiográficas de los grupos

\begin{tabular}{|c|c|c|c|c|c|}
\hline & $\begin{array}{c}\text { Total, } \\
\mathrm{n}=61(100 \%)\end{array}$ & $\begin{array}{c}\text { Controles, } \\
n=21(34 \%)\end{array}$ & $\begin{array}{c}\text { HAS, } \\
n=20(33 \%)\end{array}$ & $\begin{array}{c}\text { CoAo, } \\
n=20(33 \%)\end{array}$ & $\mathbf{p}$ \\
\hline Anillo aórtico (mm) & $21 \pm 3$ & $20 \pm 2$ & $21 \pm 4$ & $22 \pm 3$ & NS \\
\hline Seno de Valsalva (mm) & $29 \pm 5$ & $27 \pm 4$ & $29 \pm 3$ & $32 \pm 6$ & 0.04 \\
\hline Aorta ascendente (mm) & $27 \pm 4$ & $24 \pm 3$ & $27 \pm 3$ & $28 \pm 5$ & 0.01 \\
\hline Diámetro aurícula izquierda (mm) & $36 \pm 6$ & $34 \pm 4$ & $36 \pm 5$ & $38 \pm 8$ & NS \\
\hline Volumen aurícula izquierda $\mathrm{ml} / \mathrm{m}^{2} \mathrm{SC}$ & $29 \pm 12$ & $19 \pm 4$ & $33 \pm 7$ & $35 \pm 14$ & 0.0001 \\
\hline Tabique interventricular (mm) & $10 \pm 2$ & $8 \pm 1$ & $11 \pm 2$ & $11 \pm 2$ & 0.0001 \\
\hline Pared posterior del VI (mm) & $10 \pm 2$ & $8 \pm 2$ & $11 \pm 2$ & $11 \pm 2$ & 0.0001 \\
\hline Diámetro diastólico del VI (mm) & $43 \pm 6$ & $45 \pm 4$ & $40 \pm 5$ & $48 \pm 8$ & 0.0002 \\
\hline Diámetro sistólico del VI (mm) & $27 \pm 6$ & $28 \pm 4$ & $25 \pm 5$ & $32 \pm 9$ & 0.03 \\
\hline FEVI (\%) & $64 \pm 7$ & $66 \pm 3$ & $68 \pm 5$ & $58 \pm 11$ & 0.001 \\
\hline Índice de masa del VI & $88 \pm 40$ & $61 \pm 20$ & $89 \pm 20$ & $115 \pm 51$ & 0.0001 \\
\hline Relación E/A & $1.30 \pm 0.5$ & $1.4 \pm 0.30$ & $0.89 \pm 0.27$ & $1.6 \pm 0.62$ & 0.0001 \\
\hline Relación E/e' & $9 \pm 4$ & $6 \pm 1$ & $9 \pm 2$ & $12 \pm 4$ & 0.0001 \\
\hline Diámetro basal del VD (mm) & $34 \pm 5$ & $35 \pm 4$ & $35 \pm 4$ & $31 \pm 5$ & 0.02 \\
\hline TAPSE (mm) & $22 \pm 3$ & $22 \pm 3$ & $22 \pm 3$ & $23 \pm 5$ & NS \\
\hline Velocidad de onda S (cm/s) & $13 \pm 2$ & $14 \pm 1$ & $13 \pm 2$ & $13 \pm 3$ & NS \\
\hline PSAP (mmHg) & $32 \pm 8$ & $32 \pm 8$ & $26 \pm 6$ & $36 \pm 10$ & 0.001 \\
\hline Velocidad máxima transvalvular aórtica (m/s) & $1.4 \pm 0.37$ & $1.2 \pm 0.17$ & $1.3 \pm 0.24$ & $1.7 \pm 0.44$ & 0.001 \\
\hline Gradiente máximo transvalvular aórtico (mmHg) & $9 \pm 5$ & $7 \pm 2$ & $8 \pm 3$ & $13 \pm 7$ & 0.001 \\
\hline Gradiente medio transvalvular aórtico (mmHg) & $5 \pm 3$ & $3.5 \pm 0.93$ & $3.7 \pm 1.2$ & $7 \pm 4$ & 0.001 \\
\hline Velocidad máxima transcoartación (m/s) & - & - & - & $3.9 \pm 0.9$ & 0.0001 \\
\hline Gradiente máximo transcoartación (mmHg) & - & - & - & $67.03 \pm 15.89$ & 0.0001 \\
\hline
\end{tabular}

Análisis con Kruskall-Wallis. X2 y prueba exacta de Fisher.

CoAo: coartación de aorta; FEVI: fracción de expulsión del ventrículo izquierdo; HAS: hipertensión arterial sistémica; PSAP: presión sistólica de en la arteria pulmonar;

TAPSE: desplazamiento del anillo tricuspideo en sístole; VD: ventrículo derecho; VI: ventrículo izquierdo; SC: superifice corporal. 
( $p<0.0001)$, así como en el grupo de CoAo y en los hipertensos, con $r=0.45(p=0.048)$ y $r=0.47$ $(p<0.037)$, respectivamente.

La deformación radial estuvo incrementada en forma significativa solamente en los pacientes con CoAo.

La variabilidad intraobservador e interobservador se muestra en la tabla 4.

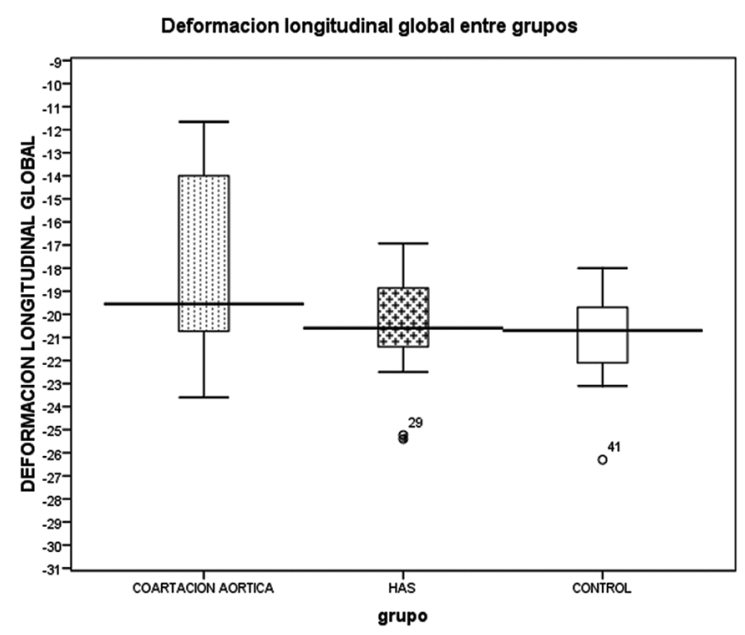

Figura 5. Gráfica de la deformación longitudinal global de los diferentes grupos de estudio.

\section{Discusión}

El seguimiento a largo plazo de los pacientes con CoAo ha demostrado que su esperanza de vida está reducida ${ }^{16,17}$. La hipertensión arterial, un importante predictor de complicaciones cardiovasculares adver$\operatorname{sas}^{17,18}$, es uno de los problemas más comunes

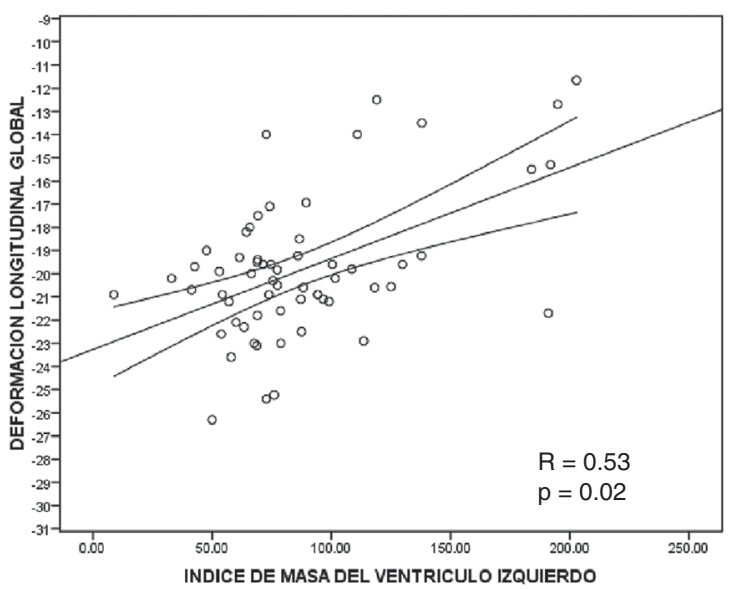

Figura 6. Correlación inversa entre la deformación longitudinal y el índice de masa del ventrículo izquierdo de los pacientes con coartación de aorta.

Tabla 3. Deformación longitudinal, radial y circunferencial, twist y rotación del ventrículo izquierdo

\begin{tabular}{|c|c|c|c|c|c|}
\hline & $\begin{array}{c}\text { Total } \\
\mathrm{n}=61(100 \%)\end{array}$ & $\begin{array}{c}\text { Controles } \\
n=21(34 \%)\end{array}$ & $\begin{array}{c}\text { HAS } \\
n=20(33 \%)\end{array}$ & $\begin{array}{c}\text { CoAo } \\
n=20(33 \%)\end{array}$ & $\mathrm{p}$ \\
\hline Deformación longitudinal global (\%) & $-20(-26,-12)$ & $-21(-26,-18)$ & $-21(-25,-17)$ & $-19(-23,-12)^{*}$ & 0.04 \\
\hline Deformación radial global (\%) & $47 \pm 13$ & $50 \pm 10$ & $39 \pm 12$ & $51 \pm 15^{*}$ & 0.004 \\
\hline Deformación circunferencial global (\%) & $-18 \pm-3.9$ & $-19 \pm-4.0$ & $-19 \pm-4.0$ & $-17 \pm 4.0$ & NS \\
\hline Rotación basal (grados) & $-5(-16,-1)$ & $-5(-11,-1.38)$ & $-6(-16,-1)$ & $-4(-16,-1.72)$ & NS \\
\hline Rotación apical (grados) & $9.6(7,29)$ & $9.0(2,26)$ & $11.0(2,29)$ & $11.0(7,24)$ & NS \\
\hline Twist (grados) & $11 \pm 8$ & $16 \pm 7$ & $17 \pm 10$ & $17 \pm 8.5$ & NS \\
\hline Torsión (grados/cm) & $2.2 \pm 1.0$ & $2.0 \pm 0.87$ & $2.4 \pm 1.3$ & $2.5 \pm 0.92$ & NS \\
\hline
\end{tabular}

CoAo: coartación de aorta; HAS: hipertensión arterial sistémica; NS: no significativo *: CoAo vs Control and HAS; ¥: CoAo vs HAS

Tabla 4. Variabilidad intraobservador e interobservador de los parámetros ecocardiográficos

\begin{tabular}{llc}
\hline Variables & Intraobservador & Interobservador \\
\hline Deformación radial & $0.816(0.315-0.950)$ & $0.776(0.532-0.934)$ \\
Deformación circunferencial & $0.875(0.603-0-961)$ & $0.830(0.487-0.961)$ \\
Deformación longitudinal & $0.99(0.964-0.970)$ & $0.987(0.953-0.997)$ \\
Fracción de expulsión del ventrículo izquierdo & $0.974(0.908-0.993)$ & $0.968(0.885-0.991)$ \\
Twist & $0.903(0.778-0.954)$ & $0.841(0.678-0.954)$ \\
Rotación & $0.883(0.626-0.964)$ & $0.754(0.478-0.927)$ \\
Índice de masa ventricular izquierda & $0.938(0.791-0.983)$ & $0.891(0.646-0.869)$ \\
\hline
\end{tabular}


observados en estos pacientes incluso después de una reparación exitosa. Inicialmente, cuando el gasto cardíaco de los ventrículos se reduce, el efecto sobre el VI puede ser mínimo debido a los mecanismos compensatorios, y los pacientes permanecen asintomáticos. Una de las principales consecuencias del remodelado ventricular relacionado con el aumento de la poscarga es el aumento del estrés parietal al final de la diástole, que puede estar presente en la $\mathrm{CoA} 0^{19,20}$. La masa ventricular izquierda es una medida reconocida para cuantificar la hipertrofia, que es un predictor independiente de eventos cardiovasculares relacionados con la $\mathrm{HAS}^{21}$. La cuantificación de los cambios en el tamaño, la masa y el desempeño del VI puede ser evaluada usando ecocardiografía bidimensional convencional. La desventaja de esta técnica en relación con la deformación miocárdica por Speckle Tracking es que es dependiente del ángulo y solo permite detectar disfunción sistólica del VI en estadios tardíos. En cambio, la deformación miocárdica del VI permite identificar la disfunción ventricular izquierda en estadios tempranos, aun cuando la función ventricular izquierda por FEVI es todavía normal.

Nuestro estudio es de gran relevancia, ya que es el primero en valorar la deformación miocárdica en pacientes adultos con CoAo y HAS no operados o no sometidos a aortoplastia con stent. Este estudio demuestra que los pacientes con CoAo y HAS tuvieron un aumento del índice de masa ventricular izquierda, el cual tuvo una correlación inversa con la deformación longitudinal global; es decir, que a mayor índice de masa ventricular izquierda, menor deformación longitudinal. En cambio, la deformación radial estuvo aumentada, lo que podría estar relacionado con un estado hiperdinámico del VI, como se ha demostrado en pacientes con CoAo que han sido sometidos a una reparación quirúrgica aparentemente exitosa ${ }^{22}$, aunque otros autores, como Shimizu, et al. ${ }^{23}$ y Palmon, et al. ${ }^{24}$, han demostrado que la hipertrofia crónica del VI se asocia con disfunción contráctil, por lo que la hipercontractilidad miocárdica que se encuentra en los pacientes con CoAo puede ser un arterfacto ${ }^{25}$. Gentles, et al. ${ }^{26}$ demostraron que el strain rate radial pico es una expresión de la contracción de la fibra de la pared media, y en presencia de poscarga baja tiene un comportamiento similar a la relación entre el estrés endocárdico y la velocidad, mostrando evidencia de un estado hipercontráctil inexplicable en los pacientes con CoAo, como nosotros hemos observado en nuestro estudio.
El twist y la rotación no mostraron diferencias estadísticamente significativas en ninguno de los grupos estudiados, a pesar de la poca variabilidad intraobservador e interobservador. Probablemente se requieran estudios con mayor número de pacientes.

Basándonos en nuestros hallazgos podemos concluir que, en los pacientes con CoAo y HAS, la deformación longitudinal global y el índice de masa ventricular izquierda se relacionan significativamente con una disfunción subclínica del VI. Estos parámetros son importantes y deben ser tomados en cuenta para normar la conducta terapéutica oportuna.

\section{Bibliografía}

1. Samánek M, Slavík Z, Zborilová B, et al. Prevalence, treatment, and outcome of heart disease in live-born children: a prospective analysis of 91,823 live-born children. Pediatr Cardiol. 1989;10:205-11.

2. Gardiner HM, Celermajer DS, Sorensen KE, et al. Arterial reactivity is significantly impaired in normotensive young adults after successful repair of aortic coarctation in childhood. Circulation. 1994;89:1745-50.

3. de Divitiis M, Pilla C, Kattenhorn M, et al. Vascular dysfunction after repair of coarctation of the aorta: impact of early surgery. Circulation. 2000;104:165-70.

4. Baumgartner H, Bonhoeffe P, De Groot NM, et al. ESC Guidelines for the management of grown-up congenital heart disease (new version 2010). The Task Force on the Management of Grown-up Congenital Heart Disease of the European Society of Cardiology (ESC) endorsed by the Association for European Paediatric Cardiology (AEPC). Eur Heart J. 2010;31:2915-57.

5. Jenkins NP, Ward C. Coarctation of the aorta: natural history and outcome after surgical treatment. QJM. 1999;92:365-71.

6. Silversides CK, Kiess M, Beauchesne L, et al. Canadian Cardiovascular Society 2009 Consensus conference on the management of adults with congenital heart disease: outflow tract obstruction, coarctation of the aorta, tetralogy of Fallot, Ebstein anomaly and Marfan's syndrome. Can J Cardiol. 2010;26:e80-e97.

7. O'Sullivan JJ, Derrick G, Darnell R. Prevalence of hypertension in children after early repair of coarctation of the aorta: a cohort study using casual and 24 hour blood pressure measurement. Heart. 2002;88:163-6.

8. Roifman I, Therrien J, lonescu-Ittu R, et al. Coarctation of the aorta and coronary artery disease: fact or fiction? Circulation. 2012;126:16-21.

9. Lang RM, Badano LP, Mor-Avi V, et al. Recommendations for cardiac chamber quantification by echocardiography in adults: an update from the American Society of Echocardiography and the European Association of Cardiovascular Imaging. J Am Soc Echocardiogr. 2015;28:1-39.

10. Voigt JU, Pedrizzetti G, Lysyansky $P$, et al. Definitions for a common standard for 2D speckle tracking echocardiography: consensus document of the EACVI/ASE/industry task force to standardize deformation imaging. J Am Soc Echocardiogr. 2015;28:183-93.

11. Gorcsan J III, Tanaka H. Echocardiographic assessment of myocardial strain. J Am Coll Cardiol. 2011;58:1401-13.

12. Collier $P$, Phelan D, Klein A. A test in context: myocardial strain measured by speckle-tracking echocardiography. J Am Coll Cardiol. 2017;69:1043-56.

13. Menting ME, van Grootel RWJ, van den Bosch AE, et al. Quantitative assessment of systolic left ventricular function with speckle-tracking echocardiography in adult patients with repaired aortic coarctation. Int J Cardiovasc Imaging. 2016;32:777-87.

14. James PA, Oparil S, Carter BL, et al. 2014 evidence-based guideline for the management of high blood pressure in adults. JAMA. 2014;311:507-20.

15. Casale PN, Devereux RB, Milner M, et al. Value of echocardiographic measurement of left ventricular mass in predicting cardiovascular morbid events in hypertensive men. Ann Intern Med. 1986;105:173-8.

16. Cohen M, Fuster V, Steele PM, et al. Coarctation of the aorta: long-term follow-up and prediction of outcome after surgical correction. Circulation. 1989;80:840-5.

17. Clarkson PM, Nicholson MR, Barratt-Boyes BG, et al. Results after repair of coarctation of the aorta beyond infancy: a 10 to 28 years follow-up with particular reference to late systemic hypertension. Am J Cardiol. 1983;81:1541-8

18. Seirafi PA, Warner KG, Geggel RL, et al. Repair of aortic coarctation during infancy minimizes the risk of late hypertension. Ann Thorac Surg. 1998;66:1378-82. 
19. Mann DL. Basic mechanisms of left ventricular remodeling: the contribution of wall stress. J Card Fail. 2004;10:S202-6.

20. Ross $\mathrm{J} \mathrm{Jr}$. Mechanisms of cardiac contraction. What roles for preload afterload and inotropic state in heart failure? Eur Heart J. 1983:4: 19-28.

21. Di Salvo G, Pacileo G, Limongelli, G, et al. Abnormal regional myocardia deformation properties and increased aortic stiffness in normotensive patients with aortic coarctation despite successful correction: an ABPM, standard echocardiography and strain rate imaging study. Clin Sci. 2007;113:259-66.

22. Crepaz R, Cemin R, Romeo $\mathrm{C}$, et al. Factors affecting left ventricular remodelling and mechanics in the long-term follow-up after successful repair of aortic coarctation. Cardiol Young. 2005;15:160-7.
23. Shimizu G, Hirota Y, Kita Y, et al. Left ventricular midwall mechanics in systemic arterial hypertension. Myocardial function is depressed in pressure-overload hypertrophy. Circulation. 1991;83:1676-84.

24. Palmon LC, Reichek N, Yeon SB, et al. Intramural myocardial shortening in hypertensive left ventricular hypertrophy with normal pump function. Circulation. 1994;89:122-31.

25. Gentles TL, Sanders SP, Colan SD. Misrepresentation of left ventricular contractile function by endocardial indexes: clinical implications after coarctation repair. Am Heart J. 2000;140:585-95.

26. Gentles TL, Cowan BR, Occleshaw CJ, et al. Midwall shortening after coarctation repair: the effect of through-plane motion on single-plane indices of left ventricular function. J Am Soc Echocardiogr. 2005;18:1131-6. 